\title{
Repeated vital sign measurements in the emergency department predict patient deterioration within 72 hours: a prospective observational study
}

Vincent M. Quinten ${ }^{1 *}$ (D), Matijs van Meurs ${ }^{2,3}$, Tycho J. Olgers ${ }^{1}$, Judith M. Vonk ${ }^{4}$, Jack J. M. Ligtenberg ${ }^{1}$ and Jan C. ter Maaten ${ }^{1}$

\begin{abstract}
Background: More than one in five patients presenting to the emergency department (ED) with (suspected) infection or sepsis deteriorate within $72 \mathrm{~h}$ from admission. Surprisingly little is known about vital signs in relation to deterioration, especially in the ED. The aim of our study was to determine whether repeated vital sign measurements in the ED can differentiate between patients who will deteriorate within $72 \mathrm{~h}$ and patients who will not deteriorate.

Methods: We performed a prospective observational study in patients presenting with (suspected) infection or sepsis to the ED of our tertiary care teaching hospital. Vital signs (heart rate, mean arterial pressure (MAP), respiratory rate and body temperature) were measured in 30-min intervals during the first $3 \mathrm{~h}$ in the ED. Primary outcome was patient deterioration within $72 \mathrm{~h}$ from admission, defined as the development of acute kidney injury, liver failure, respiratory failure, intensive care unit admission or in-hospital mortality. We performed a logistic regression analysis using a base model including age, gender and comorbidities. Thereafter, we performed separate logistic regression analyses for each vital sign using the value at admission, the change over time and its variability. For each analysis, the odds ratios (OR) and area under the receiver operator curve (AUC) were calculated.
\end{abstract}

Results: In total 106 (29.5\%) of the 359 patients deteriorated within $72 \mathrm{~h}$ from admission. Within this timeframe, 18. $3 \%$ of the patients with infection and $32.9 \%$ of the patients with sepsis at ED presentation deteriorated. Associated with deterioration were: age (OR: 1.02), history of diabetes (OR: 1.90), heart rate (OR: 1.01), MAP (OR: 0.96) and respiratory rate (OR: 1.05) at admission, changes over time of MAP (OR: 1.04) and respiratory rate (OR: 1.44) as well as the variability of the MAP (OR: 1.06). Repeated measurements of heart rate and body temperature were not associated with deterioration.

Conclusions: Repeated vital sign measurements in the ED are better at identifying patients at risk for deterioration within $72 \mathrm{~h}$ from admission than single vital sign measurements at ED admission.

Keywords: Sepsis, Accident \& emergency medicine, Patient deterioration, Vital signs

\footnotetext{
* Correspondence: v.m.quinten@umcg.nl

${ }^{1}$ Department of Emergency Medicine, University of Groningen, University Medical Center Groningen, HPC TA10, PO Box 30001, 9700 RB Groningen, The Netherlands

Full list of author information is available at the end of the article
}

(C) The Author(s). 2018 Open Access This article is distributed under the terms of the Creative Commons Attribution 4.0 International License (http://creativecommons.org/licenses/by/4.0/), which permits unrestricted use, distribution, and reproduction in any medium, provided you give appropriate credit to the original author(s) and the source, provide a link to the Creative Commons license, and indicate if changes were made. The Creative Commons Public Domain Dedication waiver (http://creativecommons.org/publicdomain/zero/1.0/) applies to the data made available in this article, unless otherwise stated. 


\section{Background}

More than one in five patients presenting to the emergency department (ED) with (suspected) infection or sepsis deteriorate within $72 \mathrm{~h}$ from admission, despite treatment [1]. Recent advances in research have improved our understanding of the pathophysiology of sepsis [2]. The adoption of surviving sepsis campaign (SSC) guidelines, increased awareness and early goal-directed therapy dramatically reduced sepsis-related mortality over the past two decades [3, 4]. However, one of the main challenges for the physician in the ED remains to determine the risk of deterioration for the individual patient [2]. The numerous sepsis-related biomarkers lack sensitivity and specificity for deterioration and are not readily available in the ED [5-7]. Despite the relative ease of measurement, surprisingly little is known about vital signs in relation to clinical outcomes, especially in the ED setting [8-11]. There is limited evidence that oxygen saturation and consciousness level at ED arrival are associated with mortality, and that heart rate and Glasgow coma scale (GCS) are associated with intensive care unit (ICU) admission [9, 11]. For all other vital signs, insufficient evidence is available $[9,11]$. The few available studies mostly studied vital signs used in triage systems or vital signs obtained at the time of ED admission $[9,12]$. Almost one third of the medical patients who arrive at the ED with normal vital signs show signs of deterioration in vital signs within $24 \mathrm{~h}$ [13]. Our pilot study in the ED showed that vital signs change significantly during the patient's stay in the ED [7]. However, surprisingly little is known on how to monitor and identify deteriorating patients in the emergency department [13]. The latest SSC guidelines recommend a thorough re-evaluation of routinely measured vital signs as parameter for response to treatment [4]. Therefore, the aim of the current study was to determine whether repeated vital sign measurements during the patient's stay in the ED can distinguish between patients who will deteriorate within $72 \mathrm{~h}$ from admission and patients who will not.

\section{Methods}

\section{Study design and setting}

This study is a predefined prospective observational study, part of the Sepsis Clinical Pathway Database (SCPD) project in our emergency department (ED). The SCPD project is a prospective cohort study of medical patients presenting to the ED with fever and/or suspected infection or sepsis. Data was collected in the ED of the University Medical Center Groningen in The Netherlands, an academic tertiary care teaching hospital with over 30,000 ED visits annually.

This study was carried out in accordance with the Declaration of Helsinki, the Dutch Agreement on Medical Treatment Act and the Dutch Personal Data Protection
Act. The Institutional Review Board of the University Medical Center Groningen ruled that the Dutch Medical Research Involving Human Subjects Act is not applicable for this study and granted a waiver (METc 2015/164). All participants provided written informed consent.

\section{Study population}

Data was collected between March 2016 and February 2017. Consecutive medical patients visiting the ED between 8 a.m. and 23 p.m. were screened for eligibility. Inclusion criteria were: (1) age of 18 years or older, (2) fever $\left(>=38{ }^{\circ} \mathrm{C}\right)$ or suspected infection or sepsis, (3) able to provide written informed consent. The clinical suspicion of infection or sepsis was judged by the coordinating internist acute medicine on duty. He/she handles all medical patient announcements from general practitioners or the emergency medical services (EMS), and medical patients that enter the ED without previous announcement. The judgement was based on information provided over the phone during the announcement, information obtained at triage and immediately after ED admission of the patient. Only patients with at least three repeated vital sign measurements during their first $3 \mathrm{~h}$ in the ED were included in the final analysis.

\section{Data collection}

The data collected in the SCPD project includes socio-demographic information, patient history, prescription drug usage, comorbidity, treatment parameters, results from routine blood analysis, questionnaires about activities of daily living, follow-up during the patient's stay in the hospital and registration of various endpoints. The data was collected by trained members of our research staff during the patient's stay in the ED and combined with data from the patient's medical record for follow-up during the patient's stay in the hospital.

For the current study, next to the data collected for all patients included in the SCPD project, we repeatedly measured vital signs in 30-min intervals during the patient's stay in de ED. These vital signs included heart rate, respiratory rate and blood pressure, measured using a Philips MP30 or MX550 bed-side patient monitor (Philips IntelliVue System with Multi-Measurement Module; Philips, Eindhoven, The Netherlands). Furthermore, the body temperature was measured using an electronic tympanic ear thermometer (Genius 2; Mountainside Medical Equipment, Marcy, New York, USA).

All patients received treatment for infection or sepsis as per our hospital's standardized protocol at the treating physician's discretion. This protocol included intravenous antibiotics, fluid resuscitation and oxygen supplementation [7]. The protocol did not change during the inclusion period and was not influenced by the patient's 
participation in the study. For patients arriving at the ED with EMS and (suspected) sepsis, treatment with fluid resuscitation and supplementary oxygen was started in the ambulance by EMS personnel according to the nationwide EMS guidelines for sepsis in The Netherlands [14]. The average time from EMS dispatch call to ED arrival is $40 \mathrm{~min}$ in The Netherlands, but actual dispatch times in this study were not measured [14]. Pre-hospital start of treatment was not influenced by the patient's participation in the study.

\section{Endpoints and definitions}

The primary endpoint was patient deterioration within $72 \mathrm{~h}$ from ED admission. We defined patient deterioration as the development of organ dysfunction, ICU admission or death during the patient's stay in the hospital. For organ dysfunction, we distinguished between acute kidney failure (AKI), liver failure and respiratory failure. AKI was defined using the Kidney Disease Improving Global Outcomes (KDIGO) criteria as an increase in serum creatinine by $26.5 \mu \mathrm{mol} / \mathrm{L}(0.3 \mathrm{mg} / \mathrm{dL})$ within $48 \mathrm{~h}$ or 1.5 times the baseline (known or presumed to have occurred within the prior 7 days) [15]. Liver failure was defined as total bilirubin level $>34.2 \mu \mathrm{mol} / \mathrm{L}$ $(2.0 \mathrm{mg} / \mathrm{dL})$ and either alkaline phosphatase or a transaminase level above twice the normal limit [16]. Respiratory failure was defined as the need for mechanical ventilation, or either hypoxemia $\left(\mathrm{PaO}_{2}<8.0 \mathrm{kPa}\right)$ or hypercapnia $\left(\mathrm{PaCO}_{2}>6.5 \mathrm{kPa}\right)$ in the arterial blood gas analysis, or a peripheral oxygen saturation $<90 \%$ when breathing ambient air or $<95 \%$ with at least $2 \mathrm{~L} / \mathrm{min}$ of oxygen supplementation [17]. In-hospital mortality was defined as all-cause mortality during the patient's stay in the hospital. The Sepsis-2 criteria (2001 international sepsis definitions conference) were used to define sepsis, severe sepsis or septic shock, i.e. two or more systemic inflammatory response syndrome criteria and suspected/ confirmed infection [18].

\section{Statistical analysis}

Continuous data were reported as median with interquartile range (IQR) and analysed using the Mann-Whitney $U$ test. Categorical data were summarized as counts with percentages and analysed using the Chi-square test.

For each vital sign and for each patient, we used the repeated measurements to estimate the linear change and variability over time. Linear change over time was estimated using individual linear regression analysis separately for each vital sign (heart rate, respiratory rate, mean arterial pressure and temperature) with the time of the measurement (in minutes) as independent variable. The resulting regression estimates for time, indicate the linear change per minute for each patient and each vital sign. The variability of each vital sign was calculated as the difference between the highest and lowest value during the first $3 \mathrm{~h}$ in the ED.

To analyse the added value of the linear change and variability over time of each vital sign as predictors for patient deterioration within $72 \mathrm{~h}$, we performed multiple logistic regression analysis. First, we constructed a base model containing age, gender and comorbidity. The added value of each vital sign to the base model was assessed using the following logistic regression analyses: (1) base model + vital sign value at admission, (2) base model + vital sign value at admission + change of the vital sign during the first $3 \mathrm{~h}$ in the ED and (3) base model + vital sign value at admission + variability of the vital sign during the first $3 \mathrm{~h}$ in the ED. For each model, the area under the receiver operator curve (AUC) was calculated using the predicted probabilities.

All statistical analyses were performed using IBM SPSS Statistics for Windows V.23.0 (IBM Corp, Armonk, New York, USA). A two-tailed $p$-value of $<0.05$ was considered significant.

\section{Results \\ Patient characteristics}

During the study period 366 patients met the inclusion criteria (Fig. 1). Seven patients were excluded because they had less than three repeated vital sign measurements in the emergency department (ED) during the first $3 \mathrm{~h}$ from admission. The remaining 359 patients were included in the final analysis. Of the 359 patients, 106 (29,5\%) patients deteriorated within $72 \mathrm{~h}$ from admission (Table 1). Patients with cardiac disease $(p=0.004)$, COPD $(p=0.047)$ or diabetes $(p=0.002)$, deteriorated more often compared to patients without these comorbidities. Malignancy (28.4\%) and organ transplant (26.7\%) were the most frequent comorbidities (Table 2).

\section{Patient deterioration}

Signs of organ failure were observed in $21.2 \%$ of the patients at ED admission (Table 3). An additional 6.1\% of the patients deteriorated in the first $24 \mathrm{~h}$ after admission. The increase in respiratory failure $(+4.2 \%)$ was the largest contributor to this deterioration. In the first $48 \mathrm{~h}$ after admission, $3.1 \%$ of the patients deteriorated to multiple organ failure. Most deterioration took place within the first $72 \mathrm{~h}$ from admission $(+8.3 \%)$, with only a small increase $(+1.7 \%)$ during the rest of the hospitalization.

In the patients who presented with infection, 14.6\% had signs of organ failure at ED admission (Table 3). An additional $3.7 \%$ of the patients with infection deteriorated in the first $24 \mathrm{~h}$ after admission. Two patients (2.4\%) required ICU admission and one patient (1.2\%) 


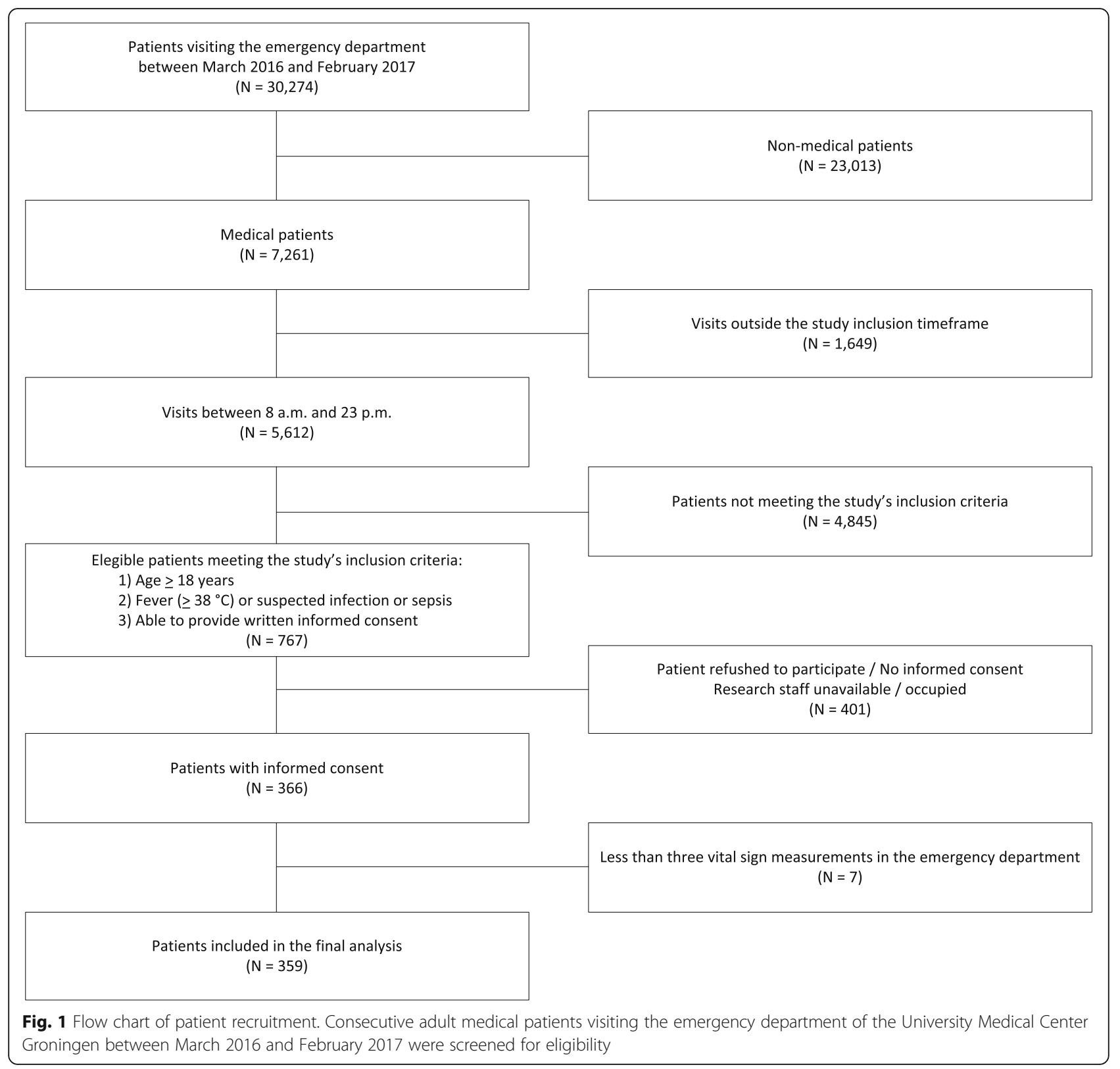

developed multiple organ failure. In the remainder of the first $72 \mathrm{~h}$, no additional patients deteriorated.

Of the patients with sepsis, $23.1 \%$ had signs of organ failure at ED admission (Table 3). Most of them had AKI (14.1\%). In the first $24 \mathrm{~h}$ after admission, an additional $6.9 \%$ of the patients with sepsis deteriorated, mostly due to respiratory failure $(+5 \%)$. An additional $1.8 \%$ of the patients deteriorated to multiple organ failure and after $48 \mathrm{~h}$ another $1.8 \%$ of the patients had developed multiple organ failure. After $72 \mathrm{~h}$, one patient had multiple organ failure in all three organ systems. During the rest of the hospitalization, only $1 \%$ of the patients deteriorated additionally. In the remainder of this article we use the first $72 \mathrm{~h}$ of admission as timeframe for patient deterioration.

\section{Age and diabetes associated with higher risk of deterioration}

The logistic regression base model for patient deterioration including age, gender and comorbidities yielded an AUC of 0.679 (Table 4). A higher age (odds ratio (OR): 1.02 / year) and a history of diabetes (OR: 1.90) were associated with a higher risk of patient deterioration. Gender and comorbidities other than diabetes were not independent predictors of deterioration. 
Table 1 Patient characteristics

\begin{tabular}{|c|c|c|c|c|}
\hline & Overall & Not deteriorated & Deteriorated & $p$ Value \\
\hline Number of patients [n (\%)] & $359(100)$ & $253(70.5)$ & $106(29.5)$ & - \\
\hline \multicolumn{5}{|l|}{ Demographics } \\
\hline Age [median (IQR)] & $63(49 ; 71)$ & $60(47 ; 70)$ & $66(56 ; 74)$ & $.001^{*}$ \\
\hline Male [n (\% of the group)] & $222(61.8)$ & $149(58.9)$ & $73(68.9)$ & .076 \\
\hline \multicolumn{5}{|l|}{ Comorbidity } \\
\hline Number of comorbidities [median (IQR)] & $1(0 ; 2)$ & $1(0 ; 2)$ & $1(1 ; 2)$ & $.001^{*}$ \\
\hline Cardiac disease [n (\% of the group)] & $66(18.4)$ & $37(14.6)$ & $29(27.4)$ & $.004^{*}$ \\
\hline COPD [n (\% of the group)] & $23(6.4)$ & $12(4.7)$ & $11(10.4)$ & $.047^{*}$ \\
\hline Diabetes [n (\%of the group)] & $63(17.5)$ & $34(13.4)$ & $29(27.4)$ & $.002^{*}$ \\
\hline Chronic kidney disease [n (\% of the group)] & $43(12.0)$ & $26(10.3)$ & $17(16.0)$ & .125 \\
\hline Chronic liver disease [n (\% of the group)] & $30(8.4)$ & $19(7.5)$ & $11(10.4)$ & .370 \\
\hline Organ transplant [n (\% of the group)] & $96(26.7)$ & $64(25.3)$ & $32(30.2)$ & .339 \\
\hline Malignancy [n (\% of the group)] & $102(28.4)$ & $77(30.4)$ & $25(23.6)$ & .189 \\
\hline None of the above [n (\% of the group)] & $98(27.3)$ & $81(32.0)$ & $17(16.0)$ & $.002^{*}$ \\
\hline \multicolumn{5}{|l|}{ Disease severity } \\
\hline Infection [n (\% of overall)] & $82(22.8)$ & $67(81.7)$ & $15(18.3)$ & $.011^{*}$ \\
\hline Sepsis [n (\% of overall)] & $277(77.2)$ & $186(67.1)$ & $91(32.9)$ & $.011^{*}$ \\
\hline \multicolumn{5}{|l|}{ Vital signs at ED admission } \\
\hline Heart rate (bpm) [median (IQR)] & $95(83.0 ; 110.0)$ & $95.0(82.0 ; 110.0)$ & $95.5(83.0 ; 110.0)$ & .262 \\
\hline Mean arterial pressure $(\mathrm{mmHg})$ [median (IQR)] & $91.7(83.3 ; 102.9)$ & $94.3(86.3 ; 103.3)$ & $85.8(73.4 ; 97.3)$ & $<.001^{*}$ \\
\hline Respiratory rate (/min) [median (IQR)] & $19.0(16.0 ; 24.0)$ & $18.0(16.0 ; 23.3)$ & $20.0(17.0 ; 25.0)$ & $.031^{*}$ \\
\hline Body temperature $\left({ }^{\circ} \mathrm{C}\right)$ [median $\left.(\mathrm{IQR})\right]$ & $37.8(37.0 ; 38.6)$ & $37.8(37.0 ; 38.6)$ & $38.0(37.0 ; 38.8)$ & .564 \\
\hline \multicolumn{5}{|l|}{ Vital sign change } \\
\hline Heart rate (bpm) [median (IQR)] & $-1.10(-2.89 ; 0.00)$ & $-1.14(-2.89 ; 0.00)$ & $-0.90(-2.93 ;-0.01)$ & .810 \\
\hline Mean arterial pressure $(\mathrm{mmHg})$ [median (IQR)] & $-0.97(-2.86 ; 0.57)$ & $-0.91(-2.71 ; 0.50)$ & $-1.18(-3.04 ; 0.84)$ & .833 \\
\hline Respiratory rate (/min) [median (IQR)] & $-0.07(-0.61 ; 0.58)$ & $-0.09(-0.69 ; 0.61)$ & $-0.07(-0.45 ; 0.56)$ & .427 \\
\hline Body temperature $\left({ }^{\circ} \mathrm{C}\right)$ [median $\left.(\mathrm{IQR})\right]$ & $-0.05(-0.17 ; 0.06)$ & $-0.05(-0.17 ; 0.06)$ & $-0.04(-0.17 ; 0.07)$ & .997 \\
\hline \multicolumn{5}{|l|}{ Vital sign variability } \\
\hline Heart rate (bpm) [median (IQR)] & $12.0(7.0 ; 20.0)$ & $12.0(7.0 ; 19.5)$ & $12.0(7.0 ; 20.5)$ & .740 \\
\hline Mean arterial pressure $(\mathrm{mmHg})$ [median (IQR)] & $15.3(9.7 ; 21.7)$ & $14.0(8.9 ; 19.5)$ & $18.2(12.6 ; 27.5)$ & $<.001^{*}$ \\
\hline Respiratory rate (/min) [median (IQR)] & $5.0(2.0 ; 8.0)$ & $4.0(2.0 ; 7.0)$ & $6.0(3.0 ; 9.8)$ & $.001^{*}$ \\
\hline Body temperature $\left({ }^{\circ} \mathrm{C}\right)$ [median (IQR)] & $0.7(0.2 ; 1.1)$ & $0.7(0.4 ; 1.1)$ & $0.7(0.4 ; 1.2)$ & .512 \\
\hline \multicolumn{5}{|l|}{ Hospital admission } \\
\hline Length of stay (days) [median (IQR)] & $4.7(0.7 ; 7.9)$ & $3.6(0.2 ; 6.2)$ & $6.7(4.1 ; 11.3)$ & $<.001^{*}$ \\
\hline \multicolumn{5}{|l|}{ Mortality } \\
\hline 28-day [n (\% of the group)] & $17(4.7)$ & $4(1.6)$ & $13(12.3)$ & $<.001^{*}$ \\
\hline 6-month [n (\% of the group)] & $44(12.3)$ & $24(9.5)$ & $20(18.9)$ & $.013^{*}$ \\
\hline
\end{tabular}

COPD: chronic obstructive pulmonary disease; ED: emergency department; IQR: interquartile range

Vital signs at ED admission are associated with deterioration

Patients who deteriorated had a lower MAP $(p<0.001)$ and a higher respiratory frequency $(p=0.03)$ at ED admission (Table 1). The base model extended with the patient's vital signs at ED admission, showed that both a higher heart rate (OR: 1.01/beat per minute; model HR-M1; AUC .683) and a higher respiratory rate (OR: 1.05/respiration per minute; model RR-M1; AUC .663) were associated with a higher risk of deterioration (Table 4, Fig. 2). A higher MAP at ED admission was associated with a lower risk of deterioration (OR: 0.96/mmHg; model MAP-M1; AUC .746). The body temperature at ED admission was not independently associated with deterioration (model BT-M1; AUC .680). 
Table 2 Study population comorbidity matrix

\begin{tabular}{|c|c|c|c|c|c|c|c|}
\hline$N=359$ & Cardiac disease & COPD & Diabetes & Chronic Kidney Disease & Chronic Liver Disease & Organ Transplant & Malignancy \\
\hline Cardiac disease & 66 & 10 & 15 & 13 & 3 & 16 & 17 \\
\hline COPD & & 23 & 3 & 1 & 1 & 4 & 5 \\
\hline Diabetes & & & 63 & 7 & 9 & 17 & 11 \\
\hline Chronic Kidney Disease & & & & 43 & 2 & 29 & 4 \\
\hline Chronic Liver Disease & & & & & 30 & 11 & 3 \\
\hline Organ Transplant & & & & & & 96 & 21 \\
\hline Malignancy & & & & & & & 102 \\
\hline
\end{tabular}

Repeated vital sign measurements improve the prediction of deterioration

Next to the vital signs at ED admission, the change and variability of the repeated vital signs measurements in the first $3 \mathrm{~h}$ in the ED were entered into the base model together with the vital signs at ED admission (Table 4, Fig. 2). An increase in MAP over time was associated with a lower risk of deterioration (OR: 0.873/unit increase; model MAP-M2; AUC .758). An increase in respiratory rate over time was associated with a higher risk of deterioration (OR: 1.441/unit increase; model RR-M2; AUC .686). The changes in heart rate and temperature were not independently associated with deterioration.
Next to the vital signs at ED admission and change over time, a higher variability in MAP (i.e. a higher range) was significantly associated with a higher risk of deterioration (OR: 1.06/mmHg; model MAP-M3; AUC .800; Table 4, Fig. 2). The variability of the other vital signs was not associated with the risk of deterioration.

\section{Discussion}

The aim of our study was to determine whether repeated vital sign measurements in the ED can identify patients with sepsis or infection that will deteriorate within $72 \mathrm{~h}$. We found an increase in MAP over time was associated

Table 3 Patient deterioration outcomes in different timeframes during the patient's stay in-hospital and divided by infection and sepsis on emergency department presentation

\begin{tabular}{|c|c|c|c|c|c|c|c|c|}
\hline & \multirow{2}{*}{$\begin{array}{l}\text { Acute } \\
\text { Kidney } \\
\text { Injury }\end{array}$} & \multirow{2}{*}{$\begin{array}{l}\text { Liver } \\
\text { failure }\end{array}$} & \multirow{2}{*}{$\begin{array}{l}\text { Respiratory } \\
\text { failure }\end{array}$} & \multicolumn{2}{|c|}{ Organ failure } & \multirow{2}{*}{$\begin{array}{l}\mathrm{ICU} \\
\text { admission }\end{array}$} & \multirow{2}{*}{$\begin{array}{l}\text { In- } \\
\text { hospital } \\
\text { mortality }\end{array}$} & \multirow[t]{2}{*}{ Deteriorated } \\
\hline & & & & Single & Multiple & & & \\
\hline \multicolumn{9}{|l|}{ Total $(\mathrm{N}=359,100.0 \%)$} \\
\hline At ED admission & $45(12.5 \%)$ & $21(5.8 \%)$ & $14(3.9 \%)$ & $72(20.1 \%)$ & $4(1.1 \%)$ & - & - & $76(21.2 \%)$ \\
\hline $24 \mathrm{~h}$ after ED admission & $51(14.2 \%)$ & $22(6.1 \%)$ & $29(8.1 \%)$ & $82(22.8 \%)$ & $10(2.8 \%)$ & $16(4.5 \%)$ & $1(0.3 \%)$ & $98(27.3 \%)$ \\
\hline $48 \mathrm{~h}$ after ED admission & 57 (15.9\%) & $23(6.4 \%)$ & $33(9.2 \%)$ & $83(23.1 \%)$ & $15(4.2 \%)$ & $18(5.0 \%)$ & $1(0.3 \%)$ & $102(28.4 \%)$ \\
\hline $72 \mathrm{~h}$ after ED admission & $60(16.7 \%)$ & $23(6.4 \%)$ & $35(9.7 \%)$ & 87 (24.2\%) & $15(4.2 \%)^{x}$ & $18(5.0 \%)$ & $3(0.8 \%)$ & $106(29.5 \%)$ \\
\hline Until hospital discharge & $70(19.5 \%)$ & $26(7.2 \%)$ & $43(12.0 \%)$ & $87(24.2 \%)$ & $24(6.7 \%)^{x x}$ & $22(6.1 \%)$ & $12(3.3 \%)$ & $112(31.2 \%)$ \\
\hline \multicolumn{9}{|l|}{ Infection $(N=82,22.8 \%)$} \\
\hline At ED admission & $6(7.3 \%)$ & $4(4.9 \%)$ & $3(3.7 \%)$ & $11(13.4 \%)$ & $1(1.2 \%)$ & - & - & $12(14.6 \%)$ \\
\hline $24 \mathrm{~h}$ after ED admission & 7 (8.5\%) & $4(4.9 \%)$ & $4(4.9 \%)$ & $11(13.4 \%)$ & $2(2.4 \%)$ & $2(2.4 \%)$ & $0(0.0 \%)$ & $15(18.3 \%)$ \\
\hline $48 \mathrm{~h}$ after ED admission & 7 (8.5\%) & $4(4.9 \%)$ & $5(6.1 \%)$ & $12(14.6 \%)$ & $2(2.4 \%)$ & $2(2.4 \%)$ & $0(0.0 \%)$ & $15(18.3 \%)$ \\
\hline $72 \mathrm{~h}$ after ED admission & $7(8.5 \%)$ & $4(4.9 \%)$ & $5(6.1 \%)$ & $12(14.6 \%)$ & $2(2.4 \%)$ & $2(2.4 \%)$ & $0(0.0 \%)$ & $15(18.3 \%)$ \\
\hline Until hospital discharge & $10(12.2 \%)$ & $6(7.3 \%)$ & $6(7.3 \%)$ & $14(17.1 \%)$ & $4(4.9 \%)$ & $3(6.7 \%)$ & $1(1.2 \%)$ & $18(22.0 \%)$ \\
\hline \multicolumn{9}{|l|}{ Sepsis $(N=277,77.2 \%)$} \\
\hline At ED admission & $39(14.1 \%)$ & $17(6.1 \%)$ & $11(4.0 \%)$ & $61(22.0 \%)$ & $3(1.1 \%)$ & - & - & $64(23.1 \%)$ \\
\hline $24 \mathrm{~h}$ after ED admission & $44(15.9 \%)$ & $18(6.5 \%)$ & $25(9.0 \%)$ & $71(25.6 \%)$ & $8(2.9 \%)$ & $14(5.1 \%)$ & $1(0.4 \%)$ & $83(30.0 \%)$ \\
\hline $48 \mathrm{~h}$ after ED admission & $50(18.1 \%)$ & 19 (6.9\%) & $28(10.1 \%)$ & $71(25.6 \%)$ & $13(4.7 \%)$ & $16(5.8 \%)$ & $1(0.4 \%)$ & $87(31.4 \%)$ \\
\hline $72 \mathrm{~h}$ after ED admission & $53(19.1 \%)$ & 19 (6.9\%) & $30(10.8 \%)$ & $75(27.1 \%)$ & $13(4.7 \%)^{x}$ & $16(5.8 \%)$ & $3(1.1 \%)$ & 91 (32.9\%) \\
\hline Until hospital discharge & $60(21.7 \%)$ & $20(7.2 \%)$ & 37 (13.4\%) & $73(26.4 \%)$ & $20(7.2 \%)^{x x}$ & $19(6.9 \%)$ & $11(4.0 \%)$ & $94(33.9 \%)$ \\
\hline
\end{tabular}

ED: emergency department; ${ }^{x}$ of which one patient with all three organ systems failing; ${ }^{x x}$ of which four patient with all three organ systems failing 
Table 4 Logistic regression models for deterioration within $72 \mathrm{~h}$ from admission based on repeated vital sign measurements with a 30-min interval during the first $3 \mathrm{~h}$ of ED admission

\begin{tabular}{|c|c|c|c|c|c|c|}
\hline & & Sig. & Odds Ratio (95\% & Model statistics & & \\
\hline & & & & Cox \& Snell R & AUC $(95 \% \mathrm{Cl})$ & $\mathrm{N}^{\mathrm{a}}$ \\
\hline Base model & leterioration within $72 \mathrm{~h}$ from adm & & & .080 & $.679(.619 ; .739)$ & $359(100 \%)$ \\
\hline Age & & $.012^{*}$ & $1.022(1.005 ; 1.039)$ & & & \\
\hline Gender (0 & ale, 1 = female) & .502 & $0.839(0.502 ; 1.402)$ & & & \\
\hline Cardiac di & & .158 & $1.544(0.845 ; 2.820)$ & & & \\
\hline COPD & & .159 & $1.906(0.777 ; 4.676)$ & & & \\
\hline Diabetes & & $.035^{*}$ & $1.902(1.048 ; 3.454)$ & & & \\
\hline Chronic k & disease & .308 & $1.475(0.699 ; 3.111)$ & & & \\
\hline Chronic li & isease & .345 & $1.493(0.650 ; 3.429)$ & & & \\
\hline Organ tra & & .245 & $1.408(0.791 ; 2.509)$ & & & \\
\hline Malignanc & & .450 & $0.807(0.463 ; 1.407)$ & & & \\
\hline Base model & heart rate & & & & & \\
\hline HR-M1. & Heart rate at admission & $.042^{*}$ & $1.013(1.000 ; 1.025)$ & .091 & $.683(.623 ; .742)$ & $359(100 \%)$ \\
\hline HR-M2. & Heart rate at admission & $.035^{*}$ & $1.015(1.001 ; 1.030)$ & .091 & $.684(.624 ; .743)$ & $358(99.7 \%)$ \\
\hline & Heart rate change & .463 & $1.039(0.938 ; 1.151)$ & & & \\
\hline HR-M3. & Heart rate at admission & .062 & $1.013(0.999 ; 1.027)$ & .091 & $.683(.624 ; .743)$ & 359 (100\%) \\
\hline & Heart rate variability & .884 & $0.998(0.977 ; 1.021)$ & & & \\
\hline Base model & mean arterial pressure & & & & & \\
\hline MAP-M1. & MAP at admission & $<.001^{*}$ & $0.955(0.937 ; 0.972)$ & .156 & $.746(.688 ; .804)$ & $357(99.4 \%)$ \\
\hline MAP-M2. & MAP at admission & $<.001^{*}$ & $0.940(0.920 ; 0.961)$ & .176 & $.758(.701 ; .815)$ & $355(98.9 \%)$ \\
\hline & MAP change & $.003^{*}$ & $0.873(0.798 ; 0.954)$ & & & \\
\hline MAP-M3. & MAP at admission & $<.001^{*}$ & $0.941(0.922 ; 0.960)$ & .223 & $.800(.750 ; .850)$ & $357(99.4 \%)$ \\
\hline & MAP variability & $<.001^{*}$ & $1.060(1.037 ; 1.084)$ & & & \\
\hline Base model & respiratory rate & & & & & \\
\hline RR-M1. & Respiratory rate at admission & $.042^{*}$ & $1.048(1.002 ; 1.097)$ & .075 & $.663(.592 ; .735)^{\mathrm{b}}$ & $267(74.4 \%)$ \\
\hline RR-M2. & Respiratory rate at admission & $.004^{*}$ & $1.086(1.027 ; 1.148)$ & .096 & $.686(.617 ; .755)^{\mathrm{b}}$ & $242(67.4 \%)$ \\
\hline & Respiratory rate change & $.018^{*}$ & $1.441(1.063 ; 1.952)$ & & & \\
\hline RR-M3. & Respiratory rate at admission & .144 & $1.022(0.988 ; 1.071)$ & .087 & $.676(.605 ; .746)^{b}$ & $267(74.4 \%)$ \\
\hline & Respiratory rate variability & .063 & $1.067(0.996 ; 1.142)$ & & & \\
\hline Base model & body temperature & & & & & \\
\hline BT-M1. & Body temperature at admission & .607 & $1.059(0.845 ; 1.319)$ & .083 & $.680(.619 ; .741)$ & $355(98.9 \%)$ \\
\hline BT-M2. & Body temperature at admission & .880 & $1.020(0.786 ; 1.324)$ & .080 & $.681(.619 ; .743)$ & $342(95.3 \%)$ \\
\hline & Body temperature change & .677 & $0.720(0.153 ; 3.385)$ & & & \\
\hline BT-M3. & Body temperature at admission & .962 & $0.994(0.790 ; 1.252)$ & .090 & $.683(.622 ; .745)$ & $355(98.9 \%)$ \\
\hline & Body temperature variability & .097 & $1.389(0.942 ; 2.049)$ & & & \\
\hline
\end{tabular}

AUC: area under the receiver operating curve; Cl: confidence interval; COPD: Chronic Obstructive Pulmonary Disease; HR: Heart rate, MAP: mean arterial pressure; RR: respiratory rate; BT: body temperature; Sig.: statistical significance; * significant result $(p<0.05)$

${ }^{a}$ Missing or observations that were constant within the measured time period are excluded from the regression model; ${ }^{b}$ the AUC of the base model only including patients with respiratory rate at admission was .638

with a lower risk of deterioration, and a higher variability of the MAP or increase in respiratory rate over time, in combination with their respective values at ED admission, were associated with patient deterioration. Inclusion of repeated MAP measurements resulted in the largest AUC (.800), whereas repeated respiratory rate measurements only slightly improved the predictive capabilities of the logistic regression model over the base model. Repeated measurements of heart rate and body temperature were not associated with patient deterioration.

Our results indicate that changes and variability of the MAP are associated with patient deterioration in ED 


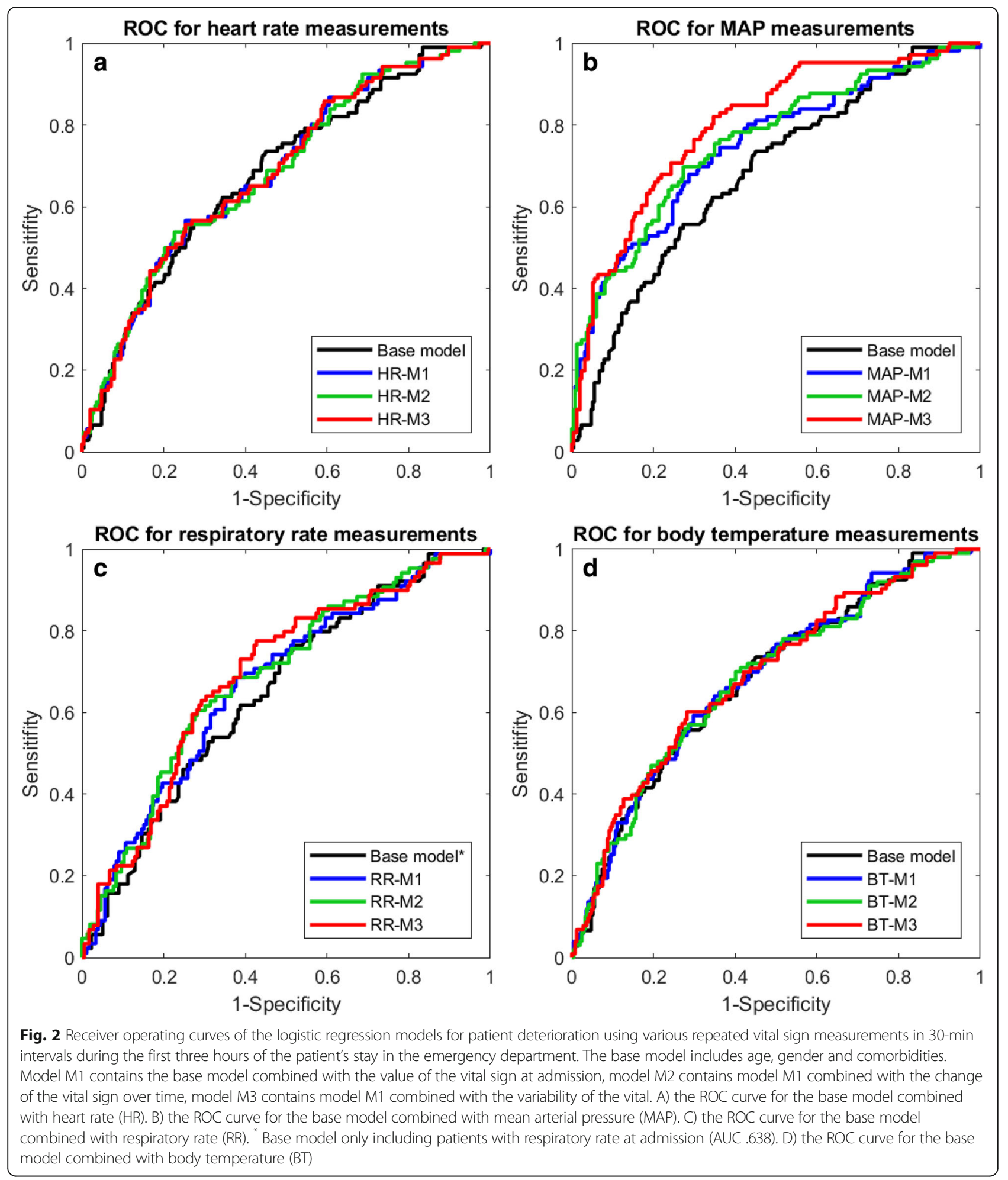

patients with infection or sepsis. This suggests that keeping a close eye on the MAP during the patients stay in the ED is important. Our study shows that this not only applies to patients with septic shock (only $1.9 \%$ of our population), as recommended by the surviving sepsis campaign (SSC) guidelines, but for all patients with sepsis or infection [4].

Apart from our earlier pilot study, little is known about repeated vital sign measurements in patients with infection or sepsis during their stay in the ED in relation 
to clinical outcomes, patient deterioration and (early) signs of organ failure. Our pilot study showed that vital signs changed significantly during the patient's stay in the ED, but did not analyse patient deterioration [7]. Henriksen et al... retrospectively found a deterioration of vital signs from the normal to abnormal range within 4$13 \mathrm{~h}$ after arrival in $31 \%$ of patients in the general ED population, leading to a four times higher 30-day mortality risk [13]. The available studies on vital signs in the ED mostly use only single measurements, mainly at triage $[9,12]$. Furthermore, these were often retrospective studies in contrast to our study. Finally, they often included the general ED population and thus a more heterogeneous population. The endpoints and cut-off values differ from study to study, most studies used mortality endpoints, several studies had ICU admission as an endpoint and only a few studies included organ failure $[8,11,13,19-22]$. The single measurements, heterogeneous patient populations and different endpoints make a direct comparison of those results with our study's results impossible. Coslovsky et al aimed to develop a prediction model for in-hospital mortality using a model with age, prolonged capillary refill, blood pressure, mechanical ventilation, oxygen saturation index, GSC and the APACHEII diagnostic category in a cohort that contained $15 \%$ patients with infection among which $7.3 \%$ with sepsis. Their model had an AUC of 0.92 , although, it should be noted that their model was based on a heterogeneous patient population, single measurements and a combination of multiple vital signs [23]. Yamamoto et al. found an association between low body temperature $\left(<36{ }^{\circ} \mathrm{C}\right)$ at ED admission and higher 30 day in-hospital mortality risk in patients with suspected sepsis [24]. In our study, we did not find an association between body temperature and deterioration. Furthermore, it should be noted that the in-hospital mortality in our study (3.3\%) is much lower than in the study of Yamamoto (9.6\%). In summary, available studies did not specifically investigate ED patients with infection or sepsis, mostly used single vital sign measurements (at triage) and primarily had mortality or ICU admission endpoints.

Early warning scores (EWS), like the national early warning score (NEWS) and many variants and related scores, are increasingly being used throughout healthcare. These EWS commonly contain a combination of various vital sign parameters, supplemented with laboratory values or other items, where each item is scored at certain thresholds. Early warning scores are mostly used as 'track-and-trigger' systems to trigger the nurse to call the physician or a rapid response team, or to predict a high risk of mortality or ICU admission [25, 26]. The many different EWS and patient populations, in which they have been validated, make it difficult to compare their performance. However, a recent review by Nannan Panday et al. showed that the NEWS score was the best to predict mortality or ICU admission in the general ED population and the modified early warning score was the best in patients with suspected infection or sepsis [25]. Their performance (AUC) was in the same range as we found for our repeated blood pressure measurements (MAP). However, it should be noted that we used only a single vital sign repeated measurement and had a composite outcome of patient deterioration, which included signs of organ dysfunction. Another recent study by Kivipuro et al. showed that the NEWS score was significantly higher before ICU admission when a patient was transferred from the ward to the ICU, compared to the NEWS score of the same patient at the ED [27]. In our hospital, modified early warning scores (MEWS) are taken at admission to the ward and thereafter three times per day. Deterioration of the MEWS score triggers an early response team. Further research is needed to clarify whether repeated vital sign measurements in combination with repeated early warning scores are useful in the detection of patient deterioration in patients with sepsis or infection.

We have shown that almost $30 \%$ of the patients presenting to the ED with suspected infection or sepsis deteriorated within $72 \mathrm{~h}$ of admission and over $28 \%$ of the patients showed signs of (multiple) organ failure despite treatment. Our results show that $18.3 \%$ of the patients with infection, $32.9 \%$ of the patients with sepsis and in total $29.5 \%$ of the patients deteriorated within $72 \mathrm{~h}$ (Table 4). Glickman et al. showed that almost $23 \%$ of patients with uncomplicated sepsis progress to severe sepsis or septic shock within $72 \mathrm{~h}$ from admission [1]. Although a direct comparison cannot be made because of a different population and different endpoints, these results clearly show that a large part of the patients with infection deteriorate in the first days in the hospital and develop (severe) sepsis. Therefore, we question whether the introduction of the recent Sepsis-3 definitions, in which infection or uncomplicated sepsis are no longer part of the sepsis severity spectrum, will lead to better patient care [28]. We would like to emphasise that it is important to properly monitor and treat all patients with infection or sepsis in the ED. Since sepsis-related mortality has dramatically reduced over the past two decades, we believe that early detection or prevention of organ failure is where the future focus of infection/sepsis research should be, since there is a lot to gain [29].

The 30-min measurement interval in the current study was arbitrarily chosen, since there is no standard on how often vital signs should be measured in the ED and only little research has been conducted on this topic. Descriptive studies in the general ED population have shown that the time between two measurements is between 67 
and $130 \mathrm{~min}$ and that a higher illness severity results in more frequent measurements $[10,30]$. We believe that these measurement intervals are not representative for patients with infection or sepsis, however, there are no specific guidelines on how often vital signs should be measured in these patients [13]. The 30-min measurement interval in our study was much more frequent than the median intervals reported by Johnson and Lambe [10, 30]. A higher measurement frequency might provide even more information about deterioration, although this might lead to a higher burden on the patient and staff. Therefore, we recommend continuous measurement of vital signs on a beat-to-beat level, preferably automated with the use of bed-side patients monitors or wearable devices [3]. Our next step, as a follow-up of this study, is to shorten the measurement interval to a beat-to-beat interval with heart rate variability (HRV) measured using bed-side patient monitors in the SepsiVit study [3]. As we have shown, a substantial number of patients deteriorate in the first days from admission. In the currently running SepsiVit study, we will extend the measurements beyond the boundaries of the ED towards the nursing wards during the first $48 \mathrm{~h}$ of hospitalization. During this period, we will investigate whether the combination of HRV with monitoring on the nursing wards can provide an early warning of patient deterioration. Such an early warning could provide a possible opportunity for intervention in the future.

\section{Strengths and limitations}

To the best of our knowledge this is the first study that prospectively investigated the relation between repeated vital sign measurements and patient deterioration in the ED in patients with infection or sepsis. We did not only use the common mortality and ICU admission endpoints, but also included signs of organ failure in our composite patient deterioration endpoint. Vital signs can be easily measured with equipment readily available in every ED. The repeated vital sign measurements in our study were obtained specifically by a trained member of our research staff, which minimized the amount of missing data. However, in spite of the prospective study design, $92(25 \%)$ respiratory rate measurements were not recorded at triage by the triage nurse. It is well-known that respiratory rate is the most frequently missing vital sign, unfortunately our study is no exception [31]. These missing respiratory rate measurements at triage limit the power of our logistic regression models that include respiratory rate (RR-M $x$; Table 4).

Another limitation of our study is that it is a single centre study in an academic tertiary care teaching hospital. This may limit the generalizability to other patient populations, especially since our population contains a high number of patients with a history of organ transplantation (Table 2). However, a history of organ transplantation was not independently associated with patient deterioration in our models (Table 4). Therefore, we believe that the specific patient population did not have a substantial influence on our results. We did not design the study to analyse combinations of multiple vital signs in our models, since we were interested in identifying which repeated vital sign measurements are helpful in predicting patient deterioration and not in the best combination of vital signs. We acknowledge that a combination of repeated vital signs may provide even more information in future studies, perhaps in combination with repeated early warning scores.

\section{Clinical implications}

We have shown that more than one in four patients presenting to the ED with suspected infection or sepsis deteriorated within $72 \mathrm{~h}$ of admission and showed signs of organ failure. These were not exclusively patients with sepsis at admission, but one in five patients that presented to the ED with infection only. Although the organ failure generally did not result in mortality, organ failure may even be preventable or treatable. Our results show that repeated vital sign measurements (especially blood pressure) at the ED is a predictor of patient deterioration and might result in a reduction of organ failure related morbidity. It is thus important to reassess patient at the ED frequently, including measurement of vital signs, as is done on the wards with early warning scores [29]. Although it is known that patient deterioration is often preceded by changes in vital signs several hours before the event, these signs are frequently missed on general wards $[25,27,32]$. At this moment, we are conducting a subsequent study (SepsiVit study) with $48 \mathrm{~h}$ of continuous vital sign measurements at the ED and on the general wards to test the hypothesis that repeated vital sign measurements at the general ward (with high frequency) is better in the prediction of patient deterioration than the currently used systems [3]. Until this information from the SepsiVit study becomes available, we assess patients at the ED frequently, including repeated vital sign measurements.

\section{Conclusions}

Repeated measurement of vital signs in the ED are better at identifying patients at risk for deterioration within $72 \mathrm{~h}$ from admission than single vital sign measurements at ED admission. Repeated measurements of MAP and respiratory rate are associated with patient deterioration. Since almost one third of patients presenting with infection or sepsis to the ED deteriorate within $72 \mathrm{~h}$, repeated vital sign measurements may be an important way to guarantee early identification of deterioration. 


\section{Abbreviations}

AKI: Acute kidney injury; AUC: Area under the receiver operator curve; BT: Body temperature; COPD: Chronic obstructive pulmonary disease; ED: Emergency department; GCS: Glasgow coma score; HR: Heart rate; HRV: Heart rate variability; ICU: Intensive care unit; KDIGO: Kidney disease improving global outcomes; MAP: Mean arterial pressure; METc: Institutional review board; OR: Odds ratio; RR: Respiratory rate; SBP: Systolic blood pressure; SCPD: Sepsis clinical pathway database; $\mathrm{SpO}_{2}$ : Peripheral oxygen saturation; SSC: Surviving sepsis campaign; USA: United States of America

\section{Acknowledgements}

The authors thank the nurses and physicians in our emergency department for their assistance during the acquisition of the data. We thank the members of the Sepsis Research Team in our emergency department for their efforts in collecting the data.

\section{Availability of data and material}

The datasets used and/or analysed during the current study are available from the corresponding author on reasonable request.

\section{Funding}

This study is funded by the emergency department of the University Medical Center Groningen. VMQ received a MD-PhD scholarship from the University of Groningen, University Medical Center Groningen for his PhD research.

\section{Authors' contributions}

VMQ drafted the study design, assisted with data acquisition, carried out data analysis and drafted the manuscript. MvM participated in the study design, assisted with data interpretation and critically revised the manuscript. TJO participated in the study design, assisted with data acquisition, and critically revised the manuscript. JMV carried out data analysis, assisted with data interpretation and critically revised the manuscript. JJML participated in the study design, assisted with data interpretation and critically revised the manuscript. JCtM participated in the study design, assisted with data interpretation, critically revised the manuscript and has given final approval of the version to be published.

\section{Ethics approval and consent to participate}

This study was carried out in accordance to the Declaration of Helsinki, the Dutch Agreement on Medical Treatment Act and the Dutch Personal Data Protection Act. The Institutional Review Board of the University Medical Center Groningen ruled that the Dutch Medical Research Involving Human Subjects Act is not applicable for this study and granted a waiver (METc 2015/164). All participants provided written informed consent.

\section{Consent for publication}

Not applicable.

\section{Competing interests}

The authors declare that they have no competing interests.

\section{Publisher's Note}

Springer Nature remains neutral with regard to jurisdictional claims in published maps and institutional affiliations.

\section{Author details}

${ }^{1}$ Department of Emergency Medicine, University of Groningen, University Medical Center Groningen, HPC TA10, PO Box 30001, 9700 RB Groningen, The Netherlands. ${ }^{2}$ Department of Critical Care, University of Groningen, University Medical Center Groningen, Groningen, The Netherlands. ${ }^{3}$ Department of Pathology and Medical Biology, Medical Biology section, University of Groningen, University Medical Center Groningen, Groningen, The Netherlands. ${ }^{4}$ Department of Epidemiology, University of Groningen, University Medical Center Groningen, Groningen, The Netherlands.

Received: 2 February 2018 Accepted: 2 July 2018

Published online: 13 July 2018

\section{References}

1. Glickman SW, Cairns CB, Otero RM, Woods CW, Tsalik EL, Langley RJ, et al. Disease progression in hemodynamically stable patients presenting to the emergency department with sepsis. Acad Emerg Med Wiley Online Library. 2010;17:383-90.

2. Buchan CA, Bravi A, Seely AJE. Variability analysis and the diagnosis, management, and treatment of sepsis. Curr Infect Dis Rep. 2012;14:512-21.

3. Quinten VM, van Meurs M, Renes MH, Ligtenberg JJM, ter Maaten JC. Protocol of the SepsiVit study: a prospective observational study to determine whether continuous heart rate variability measurement during the first 48 hours of hospitalization provides an early warning for deterioration in patients presenting with infec. BMJ Open [Internet]. British Medical Journal Publishing Group; 2017 [cited 2017 Nov 20];7:117. Available from: http://www.ncbi.nlm.nih.gov/pubmed/29151053

4. Rhodes A, Evans LE, Alhazzani W, Levy MM, Antonelli M, Ferrer R, et al. Surviving Sepsis Campaign: International Guidelines for Management of Sepsis and Septic Shock: 2016. Intensive Care Med. [Internet]. Springer Berlin Heidelberg; 2017;43:304-377. Available from: https://link.springer. com/article/10.1007/s00134-017-4683-6

5. Samraj RS, Zingarelli B, Wong HR. Role of biomarkers in sepsis care. Shock. Cincinnati. Ohio. 2013;40:358-65.

6. Pierrakos C, Vincent J-L. Sepsis biomarkers: a review. Crit Care. 2010; $14: R 15$.

7. Quinten VM, van Meurs M, Ter Maaten JC, Ligtenberg JJ. Trends in vital signs and routine biomarkers in patients with sepsis during resuscitation in the emergency department: a prospective observational pilot study. BMJ Open. 2016;6:9718.

8. Ljunggren M, Castrén M, Nordberg M, Kurland L. The association between vital signs and mortality in a retrospective cohort study of an unselected emergency department population. Scand. J. Trauma. Resusc. Emerg. Med. [Internet]. BioMed Central; 2016 [cited 2017 31];24: 21. Available from: http://www.sjtrem.com/content/24/1/21

9. Farrohknia N, Castrén $M$, Ehrenberg $A$, Lind L, Oredsson $S$, Jonsson $H$, et al. Emergency department triage scales and their components: a systematic review of the scientific evidence. Scand. J. Trauma. Resusc. Emerg. Med. [Internet]. 2011;19:42. Available from: http://sjtrem. biomedcentral.com/articles/10.1186/1757-7241-19-42

10. Lambe KR, Currey JR, Considine J. Frequency of vital sign assessment and clinical deterioration in an Australian emergency department. Australas. Emerg. Nurs. J. [Internet]. Elsevier; 2016 [cited 2017 Oct 16];19: 217-222. Available from: http://www.sciencedirect.com/science/article/ pii/S1574626716300398?via\%3Dihub

11. Hong W, Earnest A, Sultana P, Koh Z, Shahidah N, Ong MEH. How accurate are vital signs in predicting clinical outcomes in critically ill emergency department patients. Eur. J. Emerg. Med. [Internet]. Lippincott Williams \& Wilkins; 2013 [cited 2017 Oct 31];20:27-32. Available from: https://insights.ovid.com/pubmed?pmid=22198158

12. Bleyer AJ, Vidya S, Russell GB, Jones CM, Sujata L, Daeihagh $P$, et al. Longitudinal analysis of one million vital signs in patients in an academic medical center. Resuscitation [Internet]. Elsevier; 2011 [cited 2017 Nov 2];82: 1387-1392. Available from: http://www.sciencedirect.com/science/article/pii/ S0300957211004096?via\%3Dihub

13. Henriksen DP, Brabrand M, Lassen AT. Prognosis and risk factors for deterioration in patients admitted to a medical emergency department. Cleary PR, editor. PLoS One [Internet]. Public Library of Science; 2014 [cited 2017 Oct 31];9:e94649. Available from: http://dx.plos.org/10.1371/ journal.pone.0094649

14. Alam N, Oskam E, Stassen PM, van EP, van de VPM, Haak HR, et al. Prehospital antibiotics in the ambulance for sepsis: a multicentre, open label, randomised trial. Lancet Respir. Med. [Internet]. 2017; Available from: http://linkinghub.elsevier.com/retrieve/pii/S2213260017304691

15. Global Kidney Disease: Improving Outcomes (KDIGO) Acute Kidney Injury Work Group, Kellum J A, Lameire N, Aspelin P, Barsoum RS, Burdmann E A et al. KDIGO clinical practice guideline for acute kidney injury. Kidney Inter. Suppl. [Internet]. 2012:2:1-138. Available from: http://www.kdigo.org/ clinical_practice_guidelines/pdf/KDIGO\%20AKI\%20Guideline.pdf.

16. Sands $K E$, Bates DW, Lanken PN, Graman PS, Hibberd PL, Kahn KL, et al. Epidemiology of Sepsis Syndrome in 8 Academic Medical Centers. JAMA [Internet]. American Medical Association. 1997:278:234-40. Available from: http://jama.jamanetwork.com/article.aspx?doi=10.1001/ jama.1997.03550030074038

17. Burt CC, Arrowsmith JE. Respiratory failure. Surg. - Oxford Int. Ed. [Internet]. Elsevier: 2009:27:475-479. Available from: http://www. surgeryjournal.co.uk/article/S0263-9319(09)00195-1/fulltext. 
18. Levy MM, Fink MP, Marshall JC, Abraham E, Angus D, Cook D, et al. $2001 \mathrm{sccm} / \mathrm{esicm} / \mathrm{accp} / \mathrm{ats} / \mathrm{sis}$ international sepsis definitions conference. Intensive Care Med. Springer. 2003;29:530-8.

19. Kristensen AKB, Holler JG, Mikkelsen S, Hallas J, Lassen A. Systolic blood pressure and short-term mortality in the emergency department and prehospital setting: a hospital-based cohort study. Crit. Care [Internet]. BioMed Central; 2015 [cited 2017 Oct 31];19:158. Available from: http://ccforum.com/content/19/1/158

20. Barfod C, Lauritzen M, Danker J, Sölétormos G, Forberg J, Berlac P, et al. Abnormal vital signs are strong predictors for intensive care unit admission and in-hospital mortality in adults triaged in the emergency department - a prospective cohort study. Scand J Trauma Resusc Emerg Med [Internet]. BioMed Central; 2012 [cited 2017 Oct 31];20:28. Available from: http://sitrem.biomedcentral.com/articles/10.1186/1757-7241-20-28

21. Walston J, Cabrera D, Bellew S, Olive M, Lohse C, Bellolio F. Vital signs predict rapid-response team activation within twelve hours of emergency Department Admission. West. J. Emerg. Med. [Internet]. 2016 [cited 2017 Oct 31];17:324-330. Available from: http://escholarship.org/ uc/item/93r2v5j0

22. Henning DJ, Oedorf K, Day DE, Redfield CS, Huguenel CJ, Roberts JC, et al. Derivation and validation of predictive factors for clinical deterioration after admission in emergency department patients presenting with abnormal vital signs without shock. West. J Emerg Med [Internet]. California Chapter of the American Academy of Emergency Medicine (Cal/AAEM); 2015 [cited 2017 Oct 31];16:1059-1066. Available from: http://www.ncbi.nlm.nih.gov/pubmed/26759655

23. Coslovsky M, Takala J, Exadaktylos AK, Martinolli L, Merz TM. A clinical prediction model to identify patients at high risk of death in the emergency department. Intensive Care Med [Internet]. Springer Berlin Heidelberg; 2015 [cited 2017 Oct 31];41:1029-1036. Available from: http://link.springer.com/10.1007/s00134-015-3737-x

24. Yamamoto S, Yamazaki S, Shimizu T, Takeshima T, Fukuma S, Yamamoto $Y$, et al. Body temperature at the emergency department as a predictor of mortality in patients with bacterial infection. Med (Baltimore). [Internet] . 2016 [cited 2017 Oct 31];95:e3628. Available from: https://insights.ovid.com/pubmed?pmid=27227924.

25. Nannan Panday RS, Minderhoud TC, Alam N, Nanayakkara PWB. Prognostic value of early warning scores in the emergency department (ED) and acute medical unit (AMU): A narrative review [Internet]. Eur. J. Intern. Med. Elsevier; 2017 [cited 2018 Jun 20]. p. 20-31. Available from: https://www.sciencedirect.com/science/article/pii/ S0953620517303758?via\%3Dihub

26. Kyriacos U, Jelsma J, Jordan S. Monitoring vital signs using early warning scoring systems: A review of the literature. J. Nurs. Manag. [Internet]. 2011 [cited 2017 Oct 12];19:311-330. Available from: http:// doi.wiley.com/10.1111/j.1365-2834.2011.01246.x

27. Kivipuro M, Tirkkonen J, Kontula T, Solin J, Kalliomäki J, Pauniaho SL, et al. National early warning score (NEWS) in a Finnish multidisciplinary emergency department and direct vs. late admission to intensive care. Resuscitation [Internet]. Elsevier; 2018;128:164-169. Available from: https://doi.org/10.1016/.j.resuscitation.2018.05.020

28. Singer M, Deutschman CS, Seymour CW, Shankar-Hari M, Annane D, Bauer $M$, et al. The third international consensus definitions for Sepsis and septic shock (Sepsis-3). JAMA [Internet]. American Medical Association; 2016 [cited 2017 Oct 5];315:801. Available from: http:// www.ncbi.nlm.nih.gov/pubmed/26903338

29. Quinten VM, van Meurs M, Ligtenberg JJM, Ter Maaten JC. Prehospital antibiotics for sepsis: beyond mortality? Lancet Resp Med [Internet]. 2018 [cited 2018 Feb 28];6. Available from: http://www.thelancet.com/ pdfs/journals/lanres/PIIS2213-2600(18)30061-4.pdf.

30. Johnson KD, Winkelman C, Burant CJ, Dolansky M, Totten V. The Factors that affect the frequency of vital sign monitoring in the emergency department. J. Emerg. Nurs. [Internet]. Mosby; 2014 [cited 2017 Oct 31];40: 27-35. Available from: http://www.sciencedirect.com/science/article/pii/ S0099176712003923?via\%3Dihub

31. Cretikos MA, Bellomo R, Hillman K, Chen J, Finfer S, Flabouris A. Respiratory rate: the neglected vital sign. Med J Aust. 2008;188:657-9.

32. Fuhrmann L, Lippert A, Perner A, Østergaard D. Incidence, staff awareness and mortality of patients at risk on general wards. Resuscitation [Internet]. Elsevier; 2008 [cited 2018 Jun 21];77:325-330. Available from: https:/www.sciencedirect. com/science/article/pii/S030095720800052X?via\%3Dihub

\section{Ready to submit your research? Choose BMC and benefit from:}

- fast, convenient online submission

- thorough peer review by experienced researchers in your field

- rapid publication on acceptance

- support for research data, including large and complex data types

- gold Open Access which fosters wider collaboration and increased citations

- maximum visibility for your research: over $100 \mathrm{M}$ website views per year

At BMC, research is always in progress.

Learn more biomedcentral.com/submissions 